\title{
Distinct Patterns of Striatal Medium Spiny Neuron Activity during the Natural Sleep-Wake Cycle
}

\author{
Séverine Mahon, ${ }^{1 \star}$ Nicolas Vautrelle, ${ }^{2,3 \star}$ Laurent Pezard, ${ }^{4,5}$ Seán J. Slaght, ${ }^{6}$ Jean-Michel Deniau, ${ }^{1,7}$ Guy Chouvet, ${ }^{2,8}$ and \\ Stéphane Charpier ${ }^{1,7}$ \\ ${ }^{1}$ Institut National de la Santé et de la Recherche Médicale, Unité 667, Collège de France, 75231 Paris Cedex 05, France, ${ }^{2}$ Laboratoire de Neuropharmacologie \\ et de Neurochimie, Equipe d'accueil 512, Institut des Sciences Pharmaceutiques et Biologiques, Université Claude Bernard Lyon 1, 69373 Lyon, France, \\ ${ }^{3}$ Departamento de Biofisica, Instituto de Fisiologia Celular, Universidad Nacional Autonoma de Mexico, Mexico City DF, 04510 Mexico, ${ }^{4}$ Centre National de \\ la Recherche Scientifique-Unité Propre de Recherche 640, Hôpital de la Salpêtrière, 75651 Paris Cedex 13, France, 5 Université René Descartes, 92774 \\ Boulogne-Billancourt Cedex, France, ${ }^{6}$ North Middlesex University Hospital, London N18 1QX, United Kingdom, ${ }^{7}$ Université Pierre et Marie Curie, 75005 \\ Paris, France, and ${ }^{8}$ Institut National de la Santé et de la Recherche Médicale, Unité 704, Université Joseph Fourier, 38041 Grenoble, France
}

Striatal medium-sized spiny neurons (MSNs) integrate and convey information from the cerebral cortex to the output nuclei of the basal ganglia. Intracellular recordings from anesthetized animals show that MSNs undergo spontaneous transitions between hyperpolarized and depolarized states. State transitions, regarded as necessary for eliciting action potential firing in MSNs, are thought to control basal ganglia function by shaping striatal output. Here, we use an anesthetic-free rat preparation to show that the intracellular activity of MSNs is not stereotyped and depends critically on vigilance state. During slow-wave sleep, much as during anesthesia, MSNs displayed rhythmic step-like membrane potential shifts, correlated with cortical field potentials. However, wakefulness was associated with a completely different pattern of temporally disorganized depolarizing synaptic events of variable amplitude. Transitions from slow-wave sleep to wakefulness converted striatal discharge from a cyclic brisk firing to an irregular pattern of action potentials. These findings illuminate different capabilities of information processing in basal ganglia networks, suggesting in particular that a novel style of striatal computation is associated with the waking state.

Key words: striatum; electroencephalogram; intracellular recordings; up and down states; slow-wave sleep; wakefulness

\section{Introduction}

The basal ganglia are a set of interconnected subcortical nuclei implicated in a wide range of sensorimotor and cognitive functions allowing the completion of adaptive behavior (Graybiel, 2000). The striatum, the main input stage to the basal ganglia, receives and processes monosynaptic glutamatergic inputs arising from many cortical areas (McGeorge and Faull, 1989; Deniau et al., 1996; Kita, 1996; Bolam et al., 2000). The activation of GABAergic medium-sized spiny neurons (MSNs), the output neurons of the striatum, inhibits the output nuclei of basal ganglia leading to a disinhibition of thalamic and brainstem premotor nuclei (Chevalier and Deniau, 1990). The key role of MSNs in basal ganglia functions is also supported by the motor and cognitive deficits accompanying Huntington's chorea and Tourette's syndrome, which are associated with a loss or a dysfunction of MSNs (Saka and Graybiel, 2003; Levine et al., 2004).

\footnotetext{
Received Sept. 13, 2006; revised 0ct. 12, 2006; accepted 0ct. 26, 2006.

This work was supported by grants from Institut de la Santé et de la Recherche Médicale, University Pierre et Marie Curie, University Claude Bernard, and Fondation de France. We thank P. Faure, R. Miles, J. Paz, and P.--0. Polack for intellectual input throughout the redaction of this manuscript, and T. Duffau and E. Duboeuf for excellent technical assistance.

*S.M. and N.V. contributed equally to this work.

Correspondence should be addressed to Séverine Mahon, Institut National de la Santé et de la Recherche Médicale, Unité 667, Collège de France, 75231 Paris Cedex 05, France. E-mail: severine.mahon@college-de-france.fr. DOI:10.1523/JNEUROSCI.3987-06.2006

Copyright $\odot 2006$ Society for Neuroscience $\quad 0270-6474 / 06 / 2612587-09 \$ 15.00 / 0$
}

It is commonly assumed that the spontaneous intracellular activity of MSNs is characterized in vivo by rhythmic membrane potential fluctuations between a highly hyperpolarized quiescent "down" state and a depolarized "up" state associated with action potential discharge (Wilson, 1993, 1995; Wilson and Kawaguchi, 1996; Stern et al., 1997, 1998; Wickens and Wilson, 1998; Goto and O'Donnell, 2001; Tseng et al., 2001). The up state results from a synchronous excitatory synaptic barrage, arising from a large population of converging corticostriatal afferents, interacting with voltage-gated intrinsic membrane conductances, whereas the down state results from a synaptic disfacilitation associated with the activation of an inwardly rectifying potassium conductance (Wilson, 1993, 1995; Nisenbaum and Wilson, 1995; Wilson and Kawaguchi, 1996; Stern et al., 1997; Goto and O'Donnell, 2001; Mahon et al., 2001). State transitions in MSNs, which are considered as the characteristic electrical behavior of these cells, form the basis of cellular models for striatum-related physiological and pathological processes (Wilson, 1993, 1995; Houk, 1995; Stern et al., 1998; Tseng et al., 2001; Goto and O'Donnell, 2002; Kitano et al., 2002; Murer et al., 2002; Gruber et al., 2003; Carter and Sabatini, 2004).

Because of the technical difficulties inherent in intracellular recordings in alert animals, most of our knowledge on the spontaneous intracellular activity of MSNs arises from recordings from animals anesthetized with urethane and/or ketamine-xyla- 
zine (Wilson, 1993, 1995; Wilson and Kawaguchi, 1996; Stern et al., 1997, 1998; Wickens and Wilson, 1998; Goto and O'Donnell, 2001; Tseng et al., 2001). One study performed on locally anesthetized and immobilized rats, but without identification of vigilance states, suggested that MSNs could exhibit noisy irregular periods of maintained membrane depolarizations (Wilson and Groves, 1981). However, the comparison of intracellular activities of MSNs under different anesthetics indicates that the shape of spontaneous membrane potential fluctuations critically reflects the patterns of corticostriatal activity (Charpier et al., 1999; Mahon et al., 2001, 2003a), opening up the possibility that the profile of intracellular activity of MSNs could depend on the state of vigilance.

Here, we report the first intracellular recordings from MSNs during the different states of vigilance, identified by their specific patterns of electromyographic (EMG) and electroencephalographic (EEG) activity. We show that the intracellular activity of MSNs during the waking state is characterized by temporally disorganized depolarizing events, of variable amplitude and duration, which generate irregular firing patterns. In contrast, during slow-wave sleep (SWS) MSNs displayed rhythmic membrane potential fluctuations between a hyperpolarized quiescent state and a depolarized state leading to brisk action potential discharges.

\section{Materials and Methods}

The care and handling of the animals was performed in accordance with the European Communities Council Directives (86/609/EEC) and the experimental protocols were approved by an appropriate local ethical committee as well as by the French Ministry of Agriculture (03505).

Animal preparation. Experiments were conducted on four male Sprague Dawley rats (280-300 g; Charles River Laboratories, L'Arbresle, France). Surgical procedures for the fixation of the head-restraining system were performed under deep anesthesia as described previously (Souliere et al., 2000). Briefly, animals were anesthetized with an intraperitoneal injection of chloral hydrate $(400 \mathrm{mg} / \mathrm{kg}$ ) supplemented hourly (120 $\mathrm{mg} / \mathrm{kg}$ ) and mounted in a stereotaxic apparatus (Unimecanique, Epinaysur-Seine, France). Body temperature was continuously monitored and maintained at $37^{\circ} \mathrm{C}$ with an electric heating pad (Harvard Apparatus, Holliston, MA).

A focal monopolar EEG was obtained using a stainless-steel screw chronically implanted into the skull above the sensorimotor cortex (3 $\mathrm{mm}$ anterior to the bregma, $4 \mathrm{~mm}$ lateral to the midline) (Neafsey et al., 1986) and a reference electrode placed in the muscle to the opposite side of the head. Steel wires were inserted in the neck muscles to record the EMG, and all of the electrodes were connected to the pins of a miniature socket fixed to the skull with dental cement. The recording chamber consisted of a U-shaped piece of light, rigid aluminum with four headfixation bolts and was placed above the dorsal striatum and cemented to the skull. For recording sessions, rats were head-restrained by screwing the U-shaped piece to a flexible carriage (GFG, Pierre-Bénite, Rhône, France) secured to the stereotaxic frame.

Animals were then allowed to recover from surgery and anesthesia for $2 \mathrm{~d}$ and observed to be sure that the implant did not interfere with their normal behaviors. As previously reported (Souliere et al., 2000; Urbain et al., 2002), after 10-15 d of repetitive training, rats would stay still in the restraining apparatus for up to 5-6 h. During this time, they displayed normal sleep-wake cycles. The day before the first recording session, a small craniotomy $(0.5 \times 0.5 \mathrm{~mm})$ was performed, under general anesthesia (chloral hydrate, $320 \mathrm{mg} / \mathrm{kg}$, i.p.), above the striatal region of interest and the dura was removed. Typically, one recording session lasting 4-6 h was performed daily during 7-10 d. In between sessions, the recording chamber was filled with antibiotics and covered with bone wax. At the end of the experiments, rats were given a lethal dose of pentobarbital.

Electrophysiology. Intracellular recordings were obtained by inserting glass micropipettes filled with $2 \mathrm{M}$ potassium acetate $(50-70 \mathrm{M} \Omega)$ in the recording chamber. Recorded cells were located within the striatal projection field of the sensorimotor cortex $(0 \mathrm{~mm}$ to the bregma suture, $3.5-4 \mathrm{~mm}$ lateral to the midline, and $3-5.6 \mathrm{~mm}$ ventral to the brain surface) (Deniau et al., 1996).

Current-clamp recordings were made using the active bridge mode of an Axoclamp-2B amplifier (Molecular Devices, Union City, CA). Data were stored on-line on a DTR-1404 digital tape recorder (Biologic, Claix, France), and then digitized with a sampling rate of $20 \mathrm{kHz}$ (intracellular signals) or $300 \mathrm{~Hz}$ (EEG and EMG) for off-line analysis. Membrane potential values were corrected according to the tip potential recorded extracellularly immediately after the loss of the intracellular recording.

Data analysis. EEG and EMG characteristics were used to differentiate the three major states of vigilance (Timo-Iaria et al., 1970; Gottesmann, 1992). Wakefulness was distinguished by a low-voltage fast EEG activity associated with a sustained EMG tonus. Transitions between waking and SWS, referred here as drowsiness, were identified by a slowing of EEG waves, an increase in their amplitude and a decrease in the muscular tonus. SWS was recognized by high-voltage delta waves $(0.5-3 \mathrm{~Hz})$ that could be associated with spindle waves $(6-12 \mathrm{~Hz})$, and a weak EMG activity. Transitions from SWS to paradoxical sleep were characterized by a decrease in the amplitude of the slow EEG waves, the appearance of theta waves $(5-9 \mathrm{~Hz})$ and the abolition of the EMG tonus. Spectral analysis of EEG potentials was performed by applying Fast Fourier Transforms using Spike 2 (Cambridge Electronic Design, Cambridge, UK).

Histograms of membrane potential distribution were constructed for each striatal neuron. The membrane potential distribution was found to be unimodal or bimodal and could be fitted by a single or a pair of Gaussian-Laplace curves using Origin 7.0 (Microcal, Northampton, MA). In the case of bimodality, the modal value of each fit was taken as the mean membrane potential for the hyperpolarized and the depolarized state. When the membrane potential exhibited a multimodal (more than two peaks) distribution, fits were not applied and the peak values were determined from the histogram distribution.

Interspike intervals (ISIs) were measured in MSNs whose firing rate was high enough $(>1 \mathrm{~Hz})$ to allow a reliable description of ISI distribution. Because the ISIs reflecting the hyperpolarized states are several orders longer and more variable in duration than the ISIs associated with the depolarized states, we used the logarithm of the ISIs to construct ISI distribution histograms (Bhumbra and Dyball, 2004). For each MSN, histograms were normalized to the number of ISIs and then averaged between cells.

Cross-correlograms between EEG waveforms and intracellular activities (down-sampled at $300 \mathrm{~Hz}$ ) and autocorrelograms were calculated using Spike 2 routines.

Input resistance was calculated from the mean $(n \geq 12)$ steady-state voltage deflections during step hyperpolarizing current injections of weak intensity ( -0.2 to $-0.4 \mathrm{nA} ; 100-200 \mathrm{~ms}$ duration; every $1.5 \mathrm{~s})$. The membrane time constant was the time taken for the membrane potential to reach $63 \%$ of its final value. The amplitude of action potentials was calculated as the potential difference between their voltage threshold, measured as the membrane potential at which the $d V / d t$ exceeded $10 \mathrm{~V} / \mathrm{s}$ (Fricker et al., 1999; Mahon et al., 2003b) and their peak.

Time-frequency analysis of the membrane potential fluctuations was performed on down-sampled $(1 \mathrm{kHz})$ intracellular signals after spike removal and low-pass filtering $(<500 \mathrm{~Hz})$. Spikes were removed by detecting the spike threshold and approximating membrane potential values from the start to the end of the spike ( $1.3 \mathrm{~ms}$ before and $6.4 \mathrm{~ms}$ after spike threshold) by a straight line. Analysis was performed using a continuous Gabor Transform Modulus with Gaussian window (R implementation of the Swave package) (Carmona et al., 1998). The routines were written in the $\mathrm{R}$ language for statistical computing (version 2.2.1; www.R-project.org) (R Development Core Team, 2005). To quantify the frequency content of the membrane potential fluctuations within the depolarized and the hyperpolarized state, two thresholds were set at onequarter and three-quarters of the distance between the peaks of the membrane potential distribution. Membrane potentials values below the lower threshold were considered to correspond to the hyperpolarized state and those above the upper threshold to the depolarized state. The 


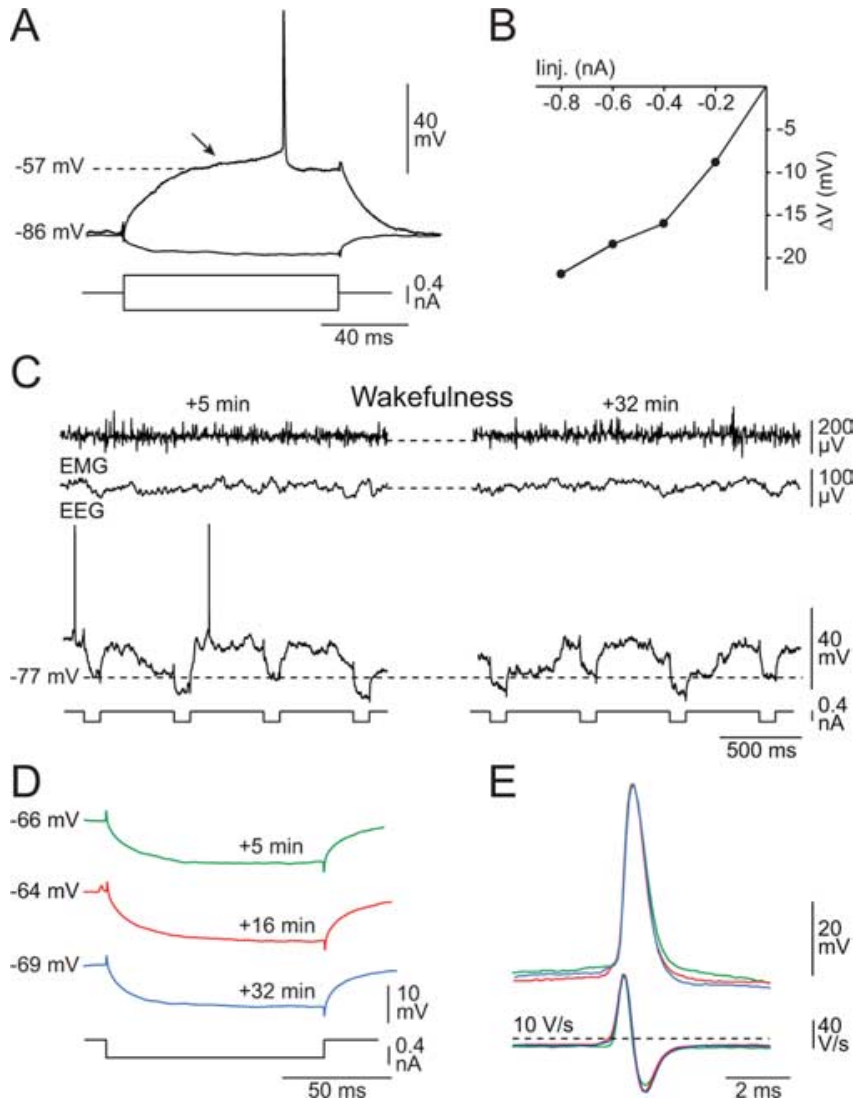

Figure 1. Electrophysiological properties of MSNs in the anesthetic-free rat. $\boldsymbol{A}$, Voltage responses of a striatal neuron (top traces) to intracellular injection of positive (single trace) and negative (average response; $n=8$ ) current pulses. Note the slow ramp depolarization (arrow) from $-60 \mathrm{mV}$ and the low input resistance $\left(R_{\text {in }}=22 \mathrm{M} \Omega\right)$. $\boldsymbol{B}$, Plot of the mean $(n=12)$ membrane potential change $(\Delta V)$ in response to negative current pulses $\left(V_{\text {inj. }}\right)$ of increasing intensity. A strong inward rectification was evident for current pulses less than $-0.4 \mathrm{nA}$. $\boldsymbol{C}-\boldsymbol{E}$, Stability of electrical membrane parameters during active waking. C, Simultaneous recordings, at different times after cell impalement, of neck EMG, cortical surface EEG, and striatal intracelIular activity (bottom) in response to repetitive injection $(2 \mathrm{~Hz})$ of negative current pulses $(0.4$ $n A)$. The fast and low amplitude EEG waves together with the phasic increases in muscular tone are characteristic of an active waking. The minimal value of spontaneous membrane potential (dashed line) remained constant throughout the recording session. $\boldsymbol{D}$, Average $(n>33)$ voltage responses to current pulses of $-0.4 \mathrm{nA}$ obtained at the indicated times after cell impalement. $\boldsymbol{E}, \mathrm{DC}$ superimposition of three spontaneous action potentials (top traces) recorded at different times (same color code as in $\boldsymbol{D}$ ) and the corresponding first-order derivative, showing the stability of spike amplitude, duration, and threshold. $\boldsymbol{A}$ and $\boldsymbol{B}-\boldsymbol{E}$ are from two different neurons.

mean frequency density spectra were calculated by averaging the squared Gabor Transform Modulus over the duration of each state (see Fig. 6C).

Numerical values are given as means \pm SD. Statistical significance was assessed by performing Student's paired or unpaired $t$ tests. The normality of distributions was tested using the Kolmogorov-Smirnov test.

\section{Results}

\section{Electrophysiological properties of MSNs in the} anesthetic-free rat

Stable intracellular recordings, of duration 2-34 min, were obtained from 15 striatal neurons located in the projection field of the sensorimotor cortex. Recorded cells displayed a relatively low input resistance $(31.9 \pm 11.3 \mathrm{M} \Omega ; n=13$ cells) (Fig. $1 A, B, D)$, a short time constant $(7.0 \pm 3.3 \mathrm{~ms} ; n=12$ cells), and hyperpolarized membrane potentials regardless of the state of vigilance (extreme value of membrane polarization, $-81.7 \pm 7.4 \mathrm{mV}$; range, -74.0 to $-100.8 \mathrm{mV} ; n=15$ cells) (Figs. $1 A, C, 2 A, 3 A, B, 4 A$,
$7 A)$. Striatal neurons exhibited, in response to threshold positive current pulses applied from rest, a slow developing ramp depolarization leading to a long-latency spike discharge (Fig. 1A). Negative current pulses of increasing intensity revealed a pronounced inward rectification evidenced by the damping of the current-induced voltage deflections (Fig. $1 B$ ). Action potentials had an amplitude of $56.5 \pm 5.0 \mathrm{mV}$ and a voltage threshold of $-51.9 \pm 2.3 \mathrm{mV}(n=15$ cells $)$. These electrophysiological properties of striatal neurons recorded during the natural sleep-wake cycle are consistent with those previously reported for MSNs recorded in vitro (Nisenbaum et al., 1994; Nisenbaum and Wilson, 1995) and from anesthetized in vivo preparations (Wilson and Kawaguchi, 1996, Wickens and Wilson, 1998; Mahon et al., 2001, 2003b).

In six MSNs, intracellular records permitted direct comparison of membrane potential fluctuations in the same cell during the sleep-wake cycle. In the remaining cells, recordings were obtained either during wakefulness $(n=6$ cells) or SWS $(n=3$ cells). Figure $1 C-E$ shows that stable intracellular recordings could be obtained even during active waking associated with phasic increases in neck muscular tone attributable to postural adjustments. Only cells exhibiting stable membrane potential (Fig. $1 C$ ), input resistance (Fig. $1 C, D$ ), spike waveform, and threshold (Fig. $1 E$ ) throughout the recording session were included in the database.

\section{Intracellular activity of MSNs during wakefulness and drowsiness}

Wakefulness was identified by a low-amplitude desynchronized EEG associated with a sustained EMG activity (Timo-Iaria et al., 1970; Gottesmann, 1992) (Fig. 2A, top). Spectral analysis performed on continuous (10-400 s) EEG records showed a dominant frequency $\sim 3-5 \mathrm{~Hz}$ associated with faster waves, up to $50 \mathrm{~Hz}$ (Fig. $2 B$, top). The corresponding intracellular striatal activity (recording duration, $8.6 \pm 10.0 \mathrm{~min} ; n=9$ cells) was characterized by sporadic depolarizing envelopes, of variable amplitude and duration, on which were superimposed high-frequency small amplitude noise-like fluctuations (Fig. 2A, top). Eight MSNs fired spontaneously, whereas one cell remained silent for the whole duration of the epoch of wakefulness. This quiescent cell did not show any distinctive electrical membrane properties, and firing could be initiated by current injection (supplemental Fig. 1, available at www.jneurosci.org as supplemental material). This suggests that, as previously reported (Sandstrom and Rebec, 2003), part of the MSN population may be silent in the awake animal. Action potentials were triggered on membrane potential fluctuations (Fig. 2A, top) with a mean firing rate of $3.3 \pm 3.5 \mathrm{~Hz}$ ( $n=8$ cells; range, $0.1-10.8 \mathrm{~Hz}$ ). ISIs were measured in MSNs whose mean firing rate exceeded $1 \mathrm{~Hz}$. In the typical example shown in Figure 2, the mean ISI was $515.6 \pm 624.4 \mathrm{~ms}$ with a corresponding coefficient of variation (CV) of 1.21. Across cells, CVs ranged from 1.21 to 1.72 (mean, $1.45 \pm 0.21 ; n=5$ cells). As shown by the autocorrelogram of spike discharge (Fig. $2 \mathrm{~A}$, bottom), the waking state was not associated with any apparent recurrent firing pattern. Consistently, MSNs exhibited positively skewed ISI distribution and the sequential ISI analysis did not reveal any particular internal structure (see Fig. $5 A$ ).

Because cortical inputs provide the main excitatory synaptic drive to MSNs (Wilson, 1993, 1995; Kincaid et al., 1998), we performed cross-correlations of simultaneously recorded cortical EEG and intracellular striatal depolarizations to test for temporal correlations between the two activities. During the waking state, there was no significant peak in the cross-correlograms (Fig. $2 B$, 
bottom), indicating a lack of temporal correlation between the desynchronized EEG and the corresponding intracellular striatal activity. This is consistent with previous findings from anesthetized animals during epochs of cortical desynchronization (Mahon et al., 2001; Kasanetz et al., 2002).

The membrane potential of MSNs during wakefulness, computed from long periods (10-60 s) of continuous recording, typically followed a unimodal, Gaussianlike $\left(r^{2}>0.9 ; n=9\right.$ cells $)$ distribution (Fig. 2C). The corresponding average membrane potential was $-69.4 \pm 9.6 \mathrm{mV}$ (range, -87.4 to $-58.0 \mathrm{mV} ; n=9$ cells) with an extreme value of membrane polarization ranging from -94.5 to $-74.0 \mathrm{mV}$ $(-81.6 \pm 7.0 \mathrm{mV} ; n=9$ cells $)$.

Waking state was occasionally followed by short, 10-20 s, periods of drowsiness ( $n=5$ periods from 5 cells) (Fig. $3 A, B$ ), which usually preceded the appearance of SWS. Drowsiness was characterized by an attenuation of EMG activity and an increase of the EEG signal power in the low frequency $(2-7 \mathrm{~Hz})$ band but without clear dominant frequency (Fig. 3C, bottom). As shown in Figure $3 D$, the transition from wakefulness to drowsiness was associated with a change from a unimodal membrane potential distribution $(-62.5 \pm 4.1 \mathrm{mV})$

(Fig. 3D, top) to a broad $(-59.0 \pm 7.1 \mathrm{mV})$, multipeak distribution (Fig. 3D, bottom).

\section{State transitions in MSNs during SWS}

SWS periods were characterized by high-amplitude lowfrequency EEG waves occasionally intermingled with spindles and a mild muscle activity without phasic contractions (TimoIaria et al., 1970; Gottesmann, 1992) (Fig. 4A, top). EEG spectral analysis indicated a high-power dominant frequency in the 1-3 $\mathrm{Hz}$ range associated with reduced power at frequencies $>10 \mathrm{~Hz}$ (Fig. $4 B$, top). Most of the MSNs $(n=7$ of 8 ) recorded during SWS showed periodic transitions in membrane potential between a hyperpolarized and a depolarized state. Transitions to the depolarized state were relatively abrupt, whereas returns to the hyperpolarized state were slower and usually exhibited an exponential-like decay (Fig. 4A, middle). Depolarized states lasted from 0.1 to $3.0 \mathrm{~s}$ and could generate single spikes or a cluster of $2-16$ action potentials (Fig. $4 A$, top). The mean firing rate in spontaneously active MSNs showed a high cell-to-cell variability (mean, $6.1 \pm 7.2 \mathrm{~Hz}$; range, $0.6-16.1 \mathrm{~Hz} ; n=6$ of 8 cells). The mean ISI for the cell illustrated in Figure $4 \mathrm{~A}$ was $287.3 \pm 512.9 \mathrm{~ms}(\mathrm{CV}, 1.78)$. Among the four computed cells, $\mathrm{CV}$ s ranged from 1.66 to 2.73 (mean, $2.0 \pm 0.49$ ). Although there was no significant difference ( $p>0.3$ ) between mean firing rates associated with SWS and wakefulness, MSNs displayed during SWS a distinct firing pattern (Fig. 4A). Consistent with the rhythmic transitions between the quiescent hyperpolarized and suprathreshold depolarized states accompanying SWS, MSNs exhibited a rhythmic brisk firing pattern (Fig. $4 A$, bottom) that was reflected in the bimodal distribution of ISIs as well as in the sequential ISI analysis (Fig. 5B). In contrast to wakefulness, crosscorrelation during SWS revealed a strong temporal coherence

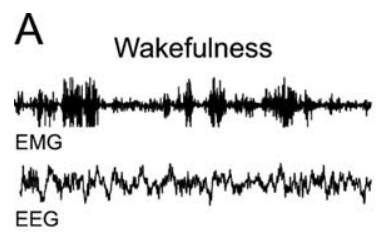

B Drowsiness
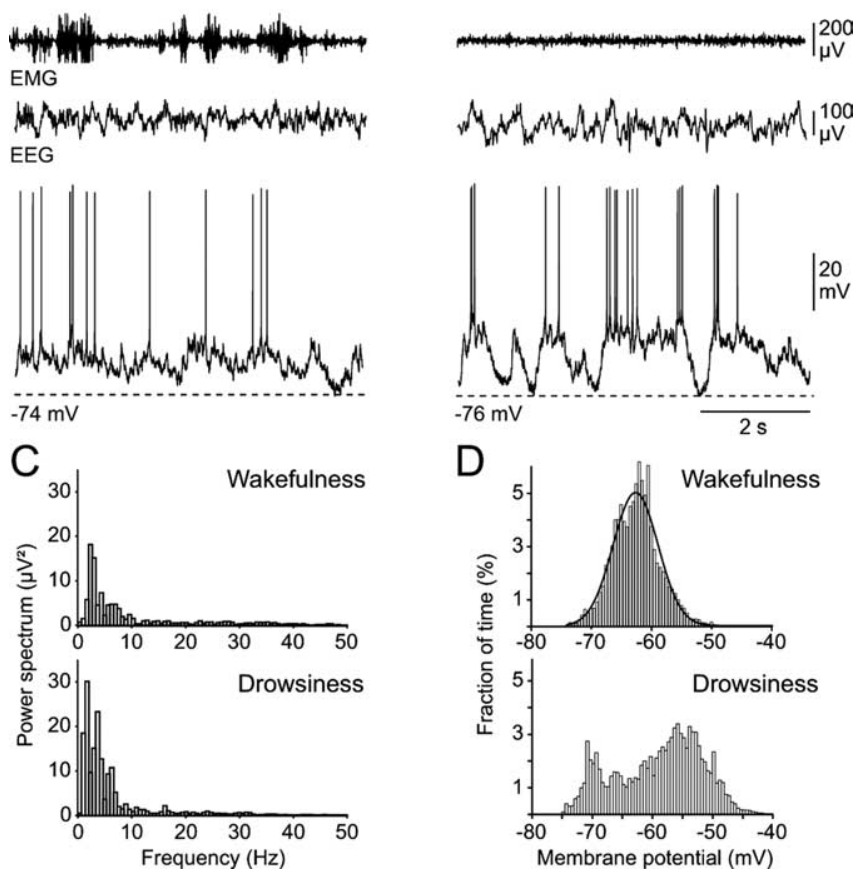

D

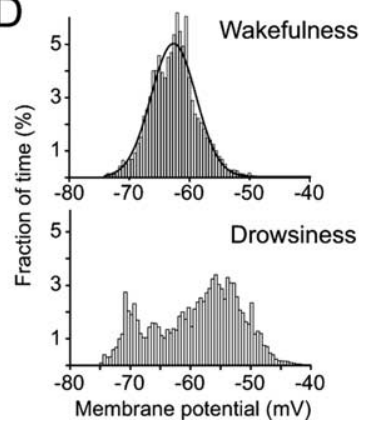

Figure 3. Alteration of MSN membrane potential distribution during drowsiness. A, B, Simultaneous recordings of intracellular striatal activity, EEG and EMG (top traces) during periods of wakefulness $(\boldsymbol{A})$ and drowsiness ( $\boldsymbol{B})$. Calibration in $\boldsymbol{B}$ applies to $\boldsymbol{A}$. $\boldsymbol{C}, \boldsymbol{D}$, Corresponding power spectra of the EEG $(\boldsymbol{C})$ and membrane potential distributions $(\boldsymbol{D})$. Note the change from a unimodal membrane potential distribution (single Gaussian fit, $r^{2}=0.97$ ) into a multipeak distribution associated with the transition from wakefulness to drowsiness. 
A
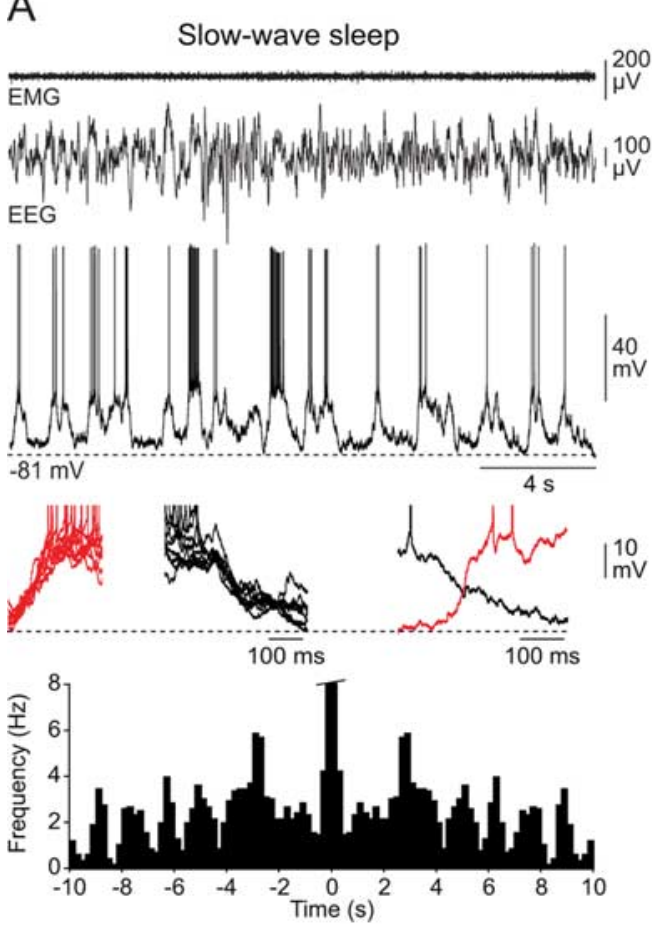

B
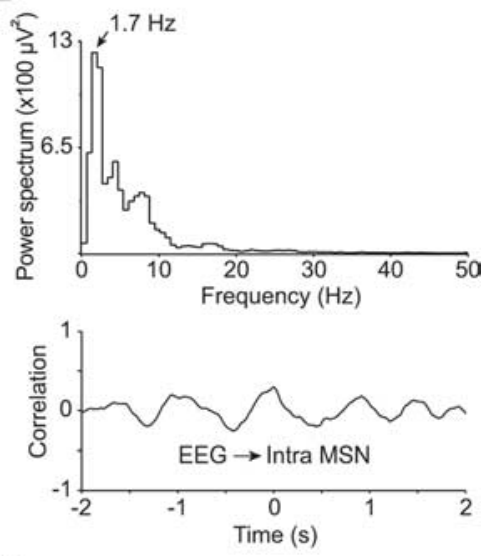

C

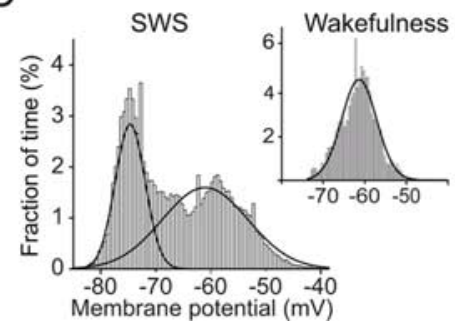

Figure 4. State transitions in MSNs during SWS. A, Top, Intracellular activity of a striatal neuron (bottom trace) during a period of SWS, together with the corresponding EEG and EMG waves. Middle, DC superimposition $(n=9$; left) of truncated records showing spontaneous transitions to the depolarized (red) and hyperpolarized (black) states. Expanded view (right) of two typical examples. Bottom, Autocorrelogram of spike discharge for the intracellular recording shown above (bin size, 0.2 s) illustrating the rhythmic firing pattern of MSNs during SWS. The central peak was truncated for clarity. $\boldsymbol{B}$, Top, Power spectrum of the EEG record shown in $A$. Bottom, EEG and intracellular activities exhibited a strong periodic correlation consistent with the optimal frequency of the EEG record ( $\sim 1-2 \mathrm{~Hz}$ ). C, Histogram of membrane potential distribution (bin size, $0.5 \mathrm{mV}$ ) calculated for a $46 \mathrm{~s}$ recording period including the recording illustrated in $A$. The histogram could be fitted by a double Gaussian $\left(r^{2}=0.97\right)$. Inset, In the same cell, the membrane potential fluctuations during a period of wakefulness exhibited a unimodal (bin size, $0.5 \mathrm{mV}$ ) distribution around a mean value of $-61.6 \pm 4.6 \mathrm{mV}$.

A
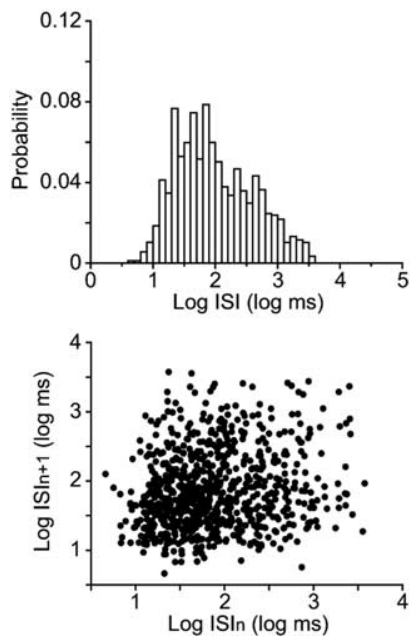

B Slow-wave sleep
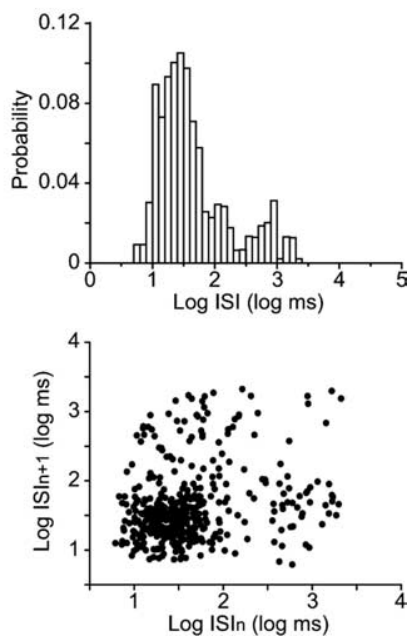

Figure 5. Comparison of firing patterns associated with wakefulness and SWS. A, Top, Averaged histogram ( $n=5$ cells) of the $\log \mid \mathrm{SI}$ distribution associated with wakefulness (bin width, $0.1 \log \mathrm{ms}$ ). Bottom, corresponding sequential analysis of the log ISIs showing the lack of serial dependence between successive intervals. $\boldsymbol{B}$, Top, Averaged histogram ( $n=4$ cells) of the $\log$ ISIs associated with SWS (bin width, $0.1 \log \mathrm{ms}$ ). Note the bimodal distribution of the ISIs. Bottom, Corresponding sequential analysis of the log ISIs. Note that firing within depolarized states was represented by a cluster of short intervals that were usually followed and preceded by longer ISIs, which correspond to the duration of hyperpolarized states.

between intracellular and EEG activity (Fig. $4 B$, bottom) at frequencies $(\sim 1 \mathrm{~Hz})$ close to those of the cortical EEG rhythm (Fig. $4 B$, top).

The two states of MSNs polarization during SWS were reflected in the bimodal distributions of membrane potential, computed from continuous records of $10-300 \mathrm{~s}$, and fitted by pairs of Gaussian curves $\left(r^{2}>0.95 ; n=6\right.$ cells) (Fig. $\left.4 C\right)$. Average membrane potentials reached during the depolarized and hyperpolarized states were $-65.4 \pm 5.8 \mathrm{mV}$ (range, -74.5 to $-59.4 \mathrm{mV} ; n=6$ cells) and $-73.9 \pm 3.5 \mathrm{mV}$ (range, -78.5 to -69.9 $\mathrm{mV} ; n=6$ cells), respectively (Fig. $4 C$ ), corresponding to a voltage difference between the two states of $8.5 \pm 3.7 \mathrm{mV}$. The profile of synaptic activity associated with the two-peak membrane potential distribution was similar to the up and down states classically described in MSNs under urethane and/or ketamine-xylazine anesthesia (Wilson, 1993; Wilson and Kawaguchi, 1996; Stern et al., 1997, 1998; Wickens and Wilson, 1998; Mahon et al., 2001; Tseng et al., 2001). In one cell, with a clearly state-dependent activity, the histogram of membrane potential followed a trimodal distribution with regularly spaced peaks at $-72.0,-64.6$, and $-57.8 \mathrm{mV}$.

The highest level of membrane polarization during SWS $(-83.1 \pm 7.8 \mathrm{mV}$; range, -100.8 to $-75.5 \mathrm{mV}$ ) as well as the mean value of membrane potential $(-70.8 \pm 7.4 \mathrm{mV})$ were similar $(p>0.5$ for both parameters) to those measured during the waking state. However, the membrane potential variance during SWS was significantly greater $(p<0.05)$ than that associated with periods of wakefulness. Figure $4 C$ shows, for one cell, the conversion of a sleep-associated bimodal membrane potential histogram (Fig. $4 C$ ) into a unimodal distribution during wakefulness (Fig. 4C, inset). Similar changes occurred in all neurons recorded during both SWS and wakefulness, suggesting that different patterns of spontaneous activity were not cell specific but rather reflected state-dependent changes throughout the MSN population.

\section{Frequency analysis of background synaptic activity}

We further characterized the temporal properties of MSN membrane potential fluctuations, presumably of synaptic origin (see Discussion), by performing time-frequency analysis of continuous records from SWS and wakefulness periods. Time-frequency analysis of the intracellular signals, after removing action potentials (Fig. $6 A, B$ ), revealed during both states of vigilance the presence of a mixture of slow and high-frequency components (Fig. $6 B, C)$. During SWS, transitions to suprathreshold depolarized states were correlated with a transient increase in power over a wide frequency band (up to $200 \mathrm{~Hz}$ ) (Fig. 6B). Fast membrane potential fluctuations were also associated with the generation of action potentials during the depolarized state (Fig. $6 A, B$ ). The power of membrane potential fluctuations was much reduced during the quiescent hyperpolarized state (Fig. $6 B, C$ ). In the same manner, random firing during wakefulness was associated 
A

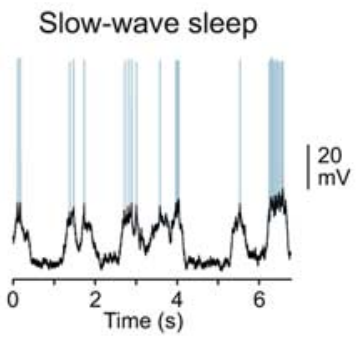

B
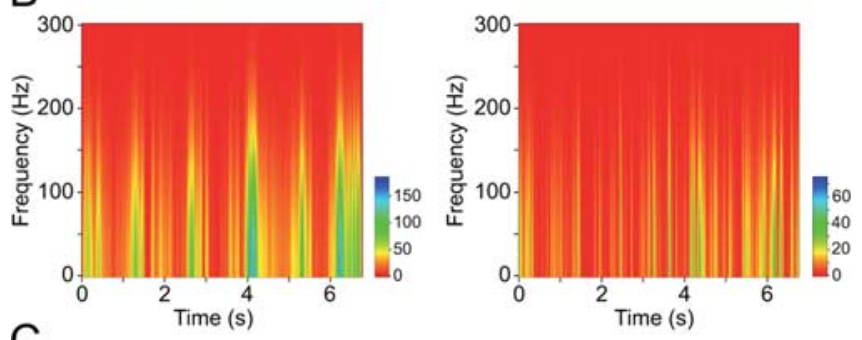

C

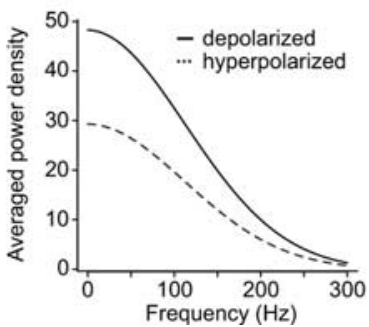

Figure 6. Frequency content analysis of striatal membrane potential fluctuations during SWS and wakefulness. $\boldsymbol{A}$, Superimposition of spontaneous intracellular activity of a striatal neuron before (light blue trace) and after spike removal (black trace) during SWS (left) and wakefulness (right). $\boldsymbol{B}$, Time-frequency analysis of the down-sampled (1 kHz) intracellular recordings shown in $\boldsymbol{A}$ (same time base). Time-frequency energy density is depicted by color scales for the time-frequency plane. Time-frequency energy density corresponds to the squared modulus of the continuous Gabor Transform computed from 0 to $300 \mathrm{~Hz}$ with $3 \mathrm{~Hz}$ intervals and a $10 \mathrm{~ms}$ Gaussian window. C, Mean Gabor spectrum power density in the $0-300$ $\mathrm{Hz}$ frequency band for the SWS-associated hyperpolarized and depolarized states. Traces in $\boldsymbol{A}$ correspond to sections of the records shown in Figures $4 A$ (SWS) and $2 A$ (wakefulness). Quantifications of the frequency content depicted in $\boldsymbol{C}$ were performed on the whole intracellular record shown in Figure $4 A$.

with an increase in power at high frequencies (Fig. $6 \mathrm{~B}$ ), suggesting that in both states of vigilance firing is exceeded by depolarizations resulting from the temporal summation of highfrequency synaptic potentials.

\section{Striatal oscillations during paradoxical sleep}

Finally, we describe a distinct form of MSN activity associated with paradoxical sleep. Epochs of paradoxical sleep are relatively short and rare in the rat (Souliere et al., 2000). We recorded striatal intracellular activity during two such episodes of duration of 100 and $250 \mathrm{~s}$ in two cells from two rats. Paradoxical sleep emerges from SWS and is characterized by the occurrence of relatively large-amplitude EEG waves in the theta range $(5-9 \mathrm{~Hz})$ (Timo-Iaria et al., 1970; Gottesmann, 1992) (Fig. 7A,B), and an almost complete loss of nuchal muscle tone (Fig. 7A). The two cells showed a remarkably similar behavior. Spontaneous activity consisted of large amplitude $(10-30 \mathrm{mV})$ subthreshold oscillations occurring at $1-2 \mathrm{~Hz}$ (Fig. $7 A, C$ ), which produced skewed unimodal membrane potential distributions around a mean of $-78.8 \pm 4.3 \mathrm{mV}$ (Fig. $7 \mathrm{D}$ ) and $-84.1 \pm 6.1 \mathrm{mV}$, respectively. The rhythmic oscillations were sculpted by the summation of presumed high-frequency depolarizing synaptic events (Fig. $7 D$, in- set). Both cells were silent at rest but current injection could induce firing suggesting that the lack of activity did not result from an alteration of intrinsic excitability (Fig. 7E).

\section{Discussion}

Our intracellular recordings from the anesthetic-free rat directly demonstrate that bistable activity is not the only spontaneous behavior generated by MSNs in physiological conditions. Rather, we showed that MSNs can exhibit various patterns of spontaneous synaptic activities providing these neurons with multiple processing capabilities. Our findings suggest that we must adjust our present conceptual frameworks for understanding the role of the striatum during physiological and pathological conditions.

\section{Membrane properties of MSNs in the alert rat}

The present study provides the first description of the intrinsic electrophysiological properties of MSNs in the anesthetic-free animal. Regardless of the state of vigilance, MSNs exhibit a highly polarized resting membrane potential, weak input resistance and time constant, and a pronounced inward rectification. Current injection from hyperpolarized potentials induced slow ramp-like depolarization, leading to a delayed spike discharge. These passive and active membrane properties, responsible for a relatively low intrinsic excitability of MSNs, are consistent with those described in vitro (Nisenbaum et al., 1994; Nisenbaum and Wilson, 1995) and in vivo under various types of anesthetics (Wilson and Kawaguchi, 1996; Wickens and Wilson, 1998; Mahon et al., 2001; Reynolds et al., 2001), where they are known to mainly result from voltage-gated potassium conductances (Wilson 1993; Nisenbaum et al. 1994; 1995; Nisenbaum and Wilson, 1995).

In addition to providing a functional identification of recorded neurons, these findings indicate that the anesthetics used in previous in vivo intracellular studies do not strongly alter the intrinsic excitability of MSNs. Moreover, they demonstrate that the distinct profiles of synaptic activity associated with the different stages of the sleep-wake cycle reflect physiological patterns of afferent activity that are lacking or hidden during anesthesia.

\section{Composite intracellular striatal activity during wakefulness}

During wakefulness, MSNs experienced continuous, slow and irregular, membrane potential fluctuations intermingled with highfrequency noise-like depolarizing events leading to a random spiking activity. This complex activity was associated with low-voltage fast EEG waves, as observed during waking in various species (Steriade, 2000; Destexhe et al., 2003). Such desynchronized EEG activity is associated in cortical pyramidal neurons with a sustained membrane depolarization that generates tonic firing (Steriade et al., 2001; Timofeev et al., 2001; Destexhe et al., 2003).

Because MSNs are innervated by thousands of corticostriatal neurons with relatively few synaptic contacts per axon (Wilson, 1995; Kincaid et al., 1998), the high-frequency noise-like membrane potential fluctuations probably originate in the uncorrelated firing of many convergent corticostriatal neurons. These small-amplitude background excitatory events triggered action potentials when concomitant with depolarizing envelopes, sculpted by the temporal summation of synaptic potentials resulting from sporadic epochs of relatively coherent cortical activity (Lopes da Silva et al., 1970; Murthy and Fetz, 1992). Because thalamic projection neurons fire tonically during alertness (Steriade, 2000), we cannot exclude that sustained discharges of glutamatergic thalamostriatal neurons (Wilson et al., 1983) contribute to the excitatory synaptic bombardment of striatal cells during wakefulness. 

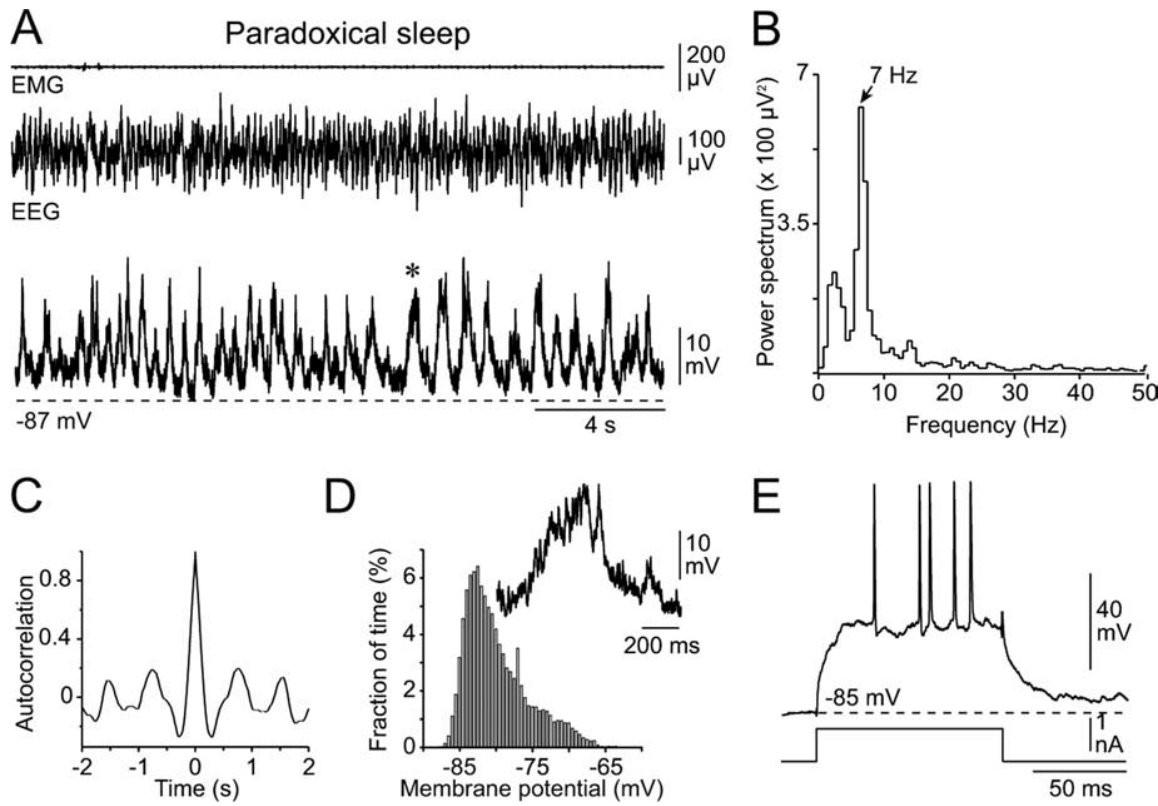

Figure 7. Subthreshold rhythmic oscillations in MSNs during paradoxical sleep. $A$, Simultaneous recordings of EMG, EEG waves and striatal cell intracellular activity. Note the complete muscular atonia. $\boldsymbol{B}$, Spectral analysis of the EEG record showing a strong periodicity at $7 \mathrm{~Hz}$. C, Autocorrelation function for the striatal activity shown in $A$ indicating a dominant frequency at $\sim 1 \mathrm{~Hz}$. Note the discrepancy between the striatal and cortical rhythms. $\boldsymbol{D}$, The unimodal membrane potential distribution (bin size, $0.5 \mathrm{mV}$ ) was skewed toward depolarized potentials. Inset, Expansion of the membrane depolarization as indicated by the asterisk in $\boldsymbol{A}$. $\boldsymbol{E}_{i}$ Voltage response of the striatal neuron (top trace) to intracellular injection of a suprathtreshold current pulse (bottom trace). Results illustrated in $\boldsymbol{A}-\boldsymbol{E}$ are from the same experiment.

We cannot exclude that the intracellular activity of MSNs in the awake head-restrained animal could slightly differ from that occurring during natural behaviors, in particular in the striatal sector related to a specific sensorimotor task. However, the present findings, showing periods of irregular repetitive discharges during active waking, are consistent with the complex firing pattern observed in freely moving animals (Kimura, 1990; Schultz et al., 2003).

Cellular mechanisms underlying MSN rhythmic depolarizations during SWS and paradoxical sleep

As in other species (Steriade, 2000), SWS was correlated with highamplitude slow EEG waves, suggesting that a large population of cortical neurons is engaged in a coherent oscillatory activity (Steriade, 2000; Steriade et al., 2001; Timofeev et al., 2001). In the anesthetic-free cat, these slow EEG potentials are correlated with the alternation of up and down states in cortical neurons (Timofeev et al., 2001). Consistently, SWS was distinguished in MSNs by the presence of rhythmic transitions between two preferred membrane potentials, a depolarized state associated with spike discharge and a quiescent hyperpolarized state, resulting in a bimodal membrane potential distribution. Given the strong temporal correlation between the rhythmic cortical field potentials and the intracellular activity of MSNs during SWS, it is very likely that the rhythmic ongoing cortical activity interacts with the active membrane properties of MSNs to determine the membrane potential shifts in striatal neurons. As proposed for the striatal up and down states during anesthesia (Nisenbaum and Wilson, 1995; Wilson, 1995; Wilson and Kawaguchi, 1996), the inwardly rectifying potassium current of MSNs should stabilize the hyperpolarized state near the potassium equilibrium potential and shunt small synaptic depolarizations. The transitions to the depolarized state may result from the temporal summation of high-frequency depolarizing events attributable to the synchronized discharge of excitatory cortical inputs. The relative stability of the membrane potential in the depolarized state may originate from interactions between the powerful depolarizing cortical synaptic drive and the activation near threshold of noninactivating and slowly inactivating voltagegated potassium currents (Nisenbaum et al., 1994; Nisenbaum and Wilson, 1995; Wilson and Kawaguchi, 1996).

The spontaneous activity of MSNs during paradoxical sleep was characterized by rhythmic $(\sim 1-2 \mathrm{~Hz})$ subthreshold oscillations sculpted by small-amplitude highfrequency depolarizing events. Given the theta rhythm $(5-9 \mathrm{~Hz})$ in the EEG activity during this state of sleep, it seems unlikely that the slow striatal oscillations reflect corticostriatal inputs. The elucidation of the origin of this activity will require records from other source of striatal afferents during paradoxical sleep.

\section{Anesthetic-dependent versus natural activity}

Up and down states, observed under urethane and/or ketamine-xylazine anesthesia, are the best described activity of striatal neurons in vivo (Wilson, 1993, 1995; Wilson and Kawaguchi, 1996; Stern et al., 1997, 1998; Wickens and Wilson, 1998; Goto and O'Donnell, 2001; Mahon et al., 2001, 2003a; Tseng et al., 2001). They are usually considered to be a unique functionally relevant activity pattern and as such shape our ideas on information processing in MSNs. Our recordings show that this two-state intracellular activity is less stereotyped during SWS than during anesthesia, with a higher temporal and voltage variability. However, our results demonstrate that the two-state activity in MSNs is not an artifact of urethane and ketamine-xylazine anesthesia but rather reflects the basic pattern of striatal activity during SWS.

We observed in SWS, at both levels of membrane polarization, a fast depolarizing voltage noise that is scarce in the anesthetized animal, particularly during the down state (Wilson and Kawaguchi, 1996; Goto and O'Donnell, 2002). The origin of this highfrequency synaptic noise during the natural hyperpolarized state is not clear. It could result from a persistent uncorrelated activity in corticostriatal and/or thalamostriatal neurons between periods of rhythmic synchrony. Alternatively, it may reflect depolarizing GABAergic events attributable to high-frequency spiking in striatal interneurons (Mallet et al., 2005) and/or extrinsic inputs arising from subcortical structures, such as the midbrain, whose activity could be altered during anesthesia (Durstewitz and Seamans, 2006). During the natural depolarized state, the increased synaptic noise might originate from corticostriatal neurons whose firing rate may be higher than under anesthesia (Steriade et al., 2001; Timofeev et al., 2001).

It is noteworthy that neurolept analgesia, associating a morphinic with a dopaminergic antagonist, induces a desynchronized EEG with low-amplitude fast waves (Mahon et al., 2001, 2003a) resembling those seen during wakefulness. Under this anesthesia, corticostriatal neurons exhibit a low-frequency irregular firing resulting in weak amplitude disorganized synaptic depolarizations in MSNs, which remain subthreshold for action potential discharge (Mahon et al., 2001, 2003a). Such a correla- 
tion between desynchronized EEG and the lack of rhythmic large-amplitude synaptic depolarizations in striatal cells is also consistent with a recent study in the urethane-anesthetized rat indicating a dramatic reduction of membrane potential fluctuations in MSNs when the rhythmic cortical ongoing activity is turned off (Kasanetz et al., 2002, 2006). During the waking state, we observed that the disorganized background synaptic activity in MSNs could generate random firing when it coincided with slow depolarizing envelopes. This irregular spiking pattern associated with unimodally distributed membrane potentials, resulting from a mixture of slow and fast membrane depolarizations, has not been described in the anesthetized animal. Although its functional significance remains to be elucidated, this complex cellular behavior provides, at least in the alert head-restrained animal, the natural intracellular activity of MSNs during waking. Contrasting with the relatively stereotyped electrical activities generated by anesthetics, our findings indicate that membrane potential fluctuations and firing patterns of central neurons are versatile and are strikingly dependent on the state of vigilance. Moreover, these results emphasize the various capabilities of computing and information processing within central networks as a function of physiological processes in which they are engaged.

\section{References}

Bhumbra GS, Dyball RE (2004) Measuring spike coding in the rat supraoptic nucleus. J Physiol (Lond) 555:281-296.

Bolam JP, Hanley JJ, Booth PA, Bevan MD (2000) Synaptic organisation of the basal ganglia. J Anat 196:527-542.

Carmona R, Hwang WL, Torresani B (1998) Practical time-frequency analysis: Gabor and wavelets transforms, with an implementation in S. San Diego: Academic.

Carter AG, Sabatini BL (2004) State-dependent calcium signaling in dendritic spines of striatal medium spiny neurons. Neuron 44:483-493.

Charpier S, Mahon S, Deniau JM (1999) In vivo induction of striatal longterm potentiation by low-frequency stimulation of the cerebral cortex. Neuroscience 91:1209-1222.

Chevalier G, Deniau JM (1990) Disinhibition as a basic process in the expression of striatal functions. Trends Neurosci 13:277-280.

Deniau JM, Menetrey A, Charpier S (1996) The lamellar organization of the rat substantia nigra pars reticulata: segregated patterns of striatal afferents and relationship to the topography of corticostriatal projections. Neuroscience 73:761-781.

Destexhe A, Rudolph M, Pare D (2003) The high-conductance state of neocortical neurons in vivo. Nat Rev Neurosci 4:739-751.

Durstewitz D, Seamans JK (2006) Beyond bistability: biophysics and temporal dynamics of working memory. Neuroscience 139:119-133.

Fricker D, Verhungen JA, Miles R (1999) Cell-attached measurements of the firing threshold of rat hippocampal neurones. J Physiol (Lond) 517:791-804.

Goto Y, O'Donnell P (2001) Network synchrony in the nucleus accumbens in vivo. J Neurosci 21:4498-4504.

Goto Y, O'Donnell P (2002) Delayed mesolimbic system alteration in a developmental animal model of schizophrenia. J Neurosci 22:9070-9077.

Gottesmann C (1992) Detection of seven sleep-waking stages in the rat. Neurosci Biobehav Rev 16:31-38.

Graybiel AM (2000) The basal ganglia. Curr Biol 10:509-511.

Gruber AJ, Solla SA, Surmeier DJ, Houk JC (2003) Modulation of striatal single units by expected reward: a spiny neuron model displaying dopamine-induced bistability. J Neurophysiol 90:1095-1114.

Houk JC (1995) Information processing in modular circuits linking basal ganglia and cerebral cortex. In: Models of information processing in the basal ganglia (Houk JC, Davies JL, Beiser DG, eds), pp 3-9. Cambridge, MA: MIT

Kasanetz F, Riquelme LA, Murer MG (2002) Disruption of the two-state membrane potential of striatal neurones during cortical desynchronisation in anaesthetised rats. J Physiol (Lond) 543:577-589.

Kasanetz F, Riquelme LA, O’Donnell P, Murer MG (2006) Turning off cor- tical ensembles stops striatal UP states and elicits phase perturbations in cortical and striatal slow oscillations in vivo. J Physiol (Lond), in press.

Kimura M (1990) Behaviorally contingent property of movement-related activity of the primate putamen. J Neurophysiol 63:1277-1296.

Kincaid AE, Zheng T, Wilson CJ (1998) Connectivity and convergence of single corticostriatal axons. J Neurosci 18:4722-4731.

Kita H (1996) Glutamatergic and gabaergic postsynaptic responses of striatal spiny neurons to intrastriatal and cortical stimulation recorded in slice preparations. Neuroscience 70:925-940.

Kitano K, Cateau H, Kaneda K, Nambu A, Takada M, Fukai T (2002) Twostate membrane potential transitions of striatal spiny neurons as evidenced by numerical simulations and electrophysiological recordings in awake monkeys. J Neurosci 22:RC230(1-6).

Levine MS, Cepeda C, Hickey MA, Fleming SM, Chesselet MF (2004) Genetic mouse models of Huntington's and Parkinson's diseases: illuminating but imperfect. Trends Neurosci 27:691-697.

Lopes da Silva FH, van Rotterdam A, Storm van Leeuwen W, Tielen AM (1970) Dynamic characteristics of visual evoked potentials in the dog. II. Beta frequency selectivity in evoked potentials and background activity. Electroencephalogr Clin Neurophysiol 29:260-268.

Mahon S, Deniau JM, Charpier S (2001) Relationship between EEG potentials and intracellular activity of striatal and cortico-striatal neurons: an in vivo study under different anesthetics. Cereb Cortex 11:360-373.

Mahon S, Deniau JM, Charpier S (2003a) Various synaptic activities and firing patterns in cortico-striatal and striatal neurons in vivo. J Physiol (Paris) 97:557-566.

Mahon S, Casassus G, Mulle C, Charpier S (2003b) Spike-dependent intrinsic plasticity increases firing probability in rat striatal neurons in vivo. J Physiol (Lond) 550:947-959.

Mallet N, Le Moine C, Charpier S, Gonon F (2005) Feedforward inhibition of projection neurons by fast spiking GABA interneurons in the rat striatum in vivo. J Neurosci 25:3857-3869.

McGeorge AJ, Faull RLM (1989) The organization from the projection from the cerebral cortex to the striatum in the rat. Neuroscience 29:503-537.

Murer MG, Tseng KY, Kasanetz F, Belluscio M, Riquelme LA (2002) Brain oscillations, medium spiny neurons, and dopamine. Cell Mol Neurobiol 22:611-632.

Murthy VN, Fetz EE (1992) Coherent 25- to 35-Hz oscillations in the sensorimotor cortex of awake behaving monkeys. Proc Natl Acad Sci USA 89:5670-5674.

Neafsey EJ, Bold EL, Haas G, Hurley-Gius KM, Quirk G, Sievert CF, Terreberry RR (1986) The organization of the rat motor cortex: a microstimulation mapping study. Brain Res Rev 11:77-96.

Nisenbaum ES, Wilson CJ (1995) Potassium current responsible for inward and outward rectification in rat neostriatal spiny projection neurons. J Neurosci 15:4449-4463.

Nisenbaum ES, Xu ZC, Wilson CJ (1994) Contribution of a slowly inactivating potassium current to the transition to firing of neostriatal spiny projections neurons. J Neurophysiol 71:1174-1189.

R Development Core Team (2005) R: a language and environment for statistical computing. Vienna: R Foundation for Statistical Computing.

Reynolds JN, Hyland BI, Wickens JR (2001) A cellular mechanism of reward-related learning. Nature 413:67-70.

Saka E, Graybiel AM (2003) Pathophysiology of Tourette's syndrome: striatal pathways revisited. Brain Dev 25:S15-S19.

Sandstrom MI, Rebec GV (2003) Characterization of striatal activity in conscious rats: contribution of NMDA and AMPA/kainate receptors to both spontaneous and glutamate-driven firing. Synapse 47:91-100.

Schultz W, Tremblay L, Hollerman JR (2003) Changes in behavior-related neuronal activity in the striatum during learning. Trends Neurosci 26:321-328.

Souliere F, Urbain N, Gervasoni D, Schmitt P, Guillemort C, Fort P, Renaud B, Luppi PH, Chouvet G (2000) Single-unit and polygraphic recordings associated with systemic or local pharmacology: a multi-purpose stereotaxic approach for the awake, anaesthetic-free, and head-restrained rat. J Neurosci Res 61:88-100.

Steriade M (2000) Corticothalamic resonance, states of vigilance and mentation. Neuroscience 101:243-276.

Steriade M, Timofeev I, Grenier F (2001) Natural waking and sleep states: a view from inside neocortical neurons. J Neurophysiol 85:1969-1985.

Stern EA, Kincaid AE, Wilson CJ (1997) Spontaneous subthreshold mem- 
brane potential fluctuations and action potential variability of rat corticostriatal and striatal neurons in vivo. J Neurophysiol 77:1697-1715.

Stern EA, Jaeger D, Wilson CJ (1998) Membrane potential synchrony of simultaneously recorded striatal spiny neurons in vivo. Nature 394:475-478.

Timofeev I, Grenier F, Steriade M (2001) Disfacilitation and active inhibition in the neocortex during the natural sleep-wake cycle: an intracellular study. Proc Natl Acad Sci USA 98:1924-1929.

Timo-Iaria C, Negrao N, Schmidek WR, Hoshino K, Lobato de Menezes CE, Leme da Rocha T (1970) Phases and states of sleep in the rat. Physiol Behav 5:1057-1062.

Tseng KY, Kasanetz F, Kargieman L, Riquelme LA, Murer MG (2001) Cortical slow oscillatory activity is reflected in the membrane potential and spike trains of striatal neurons in rats with chronic nigrostriatal lesions. J Neurosci 21:6430-6439.

Urbain N, Rentero N, Gervasoni D, Renaud B, Chouvet G (2002) The switch of subthalamic neurons from an irregular to a bursting pattern does not solely depend on their GABAergic inputs in the anesthetic-free rat. J Neurosci 22:8665-8675.
Wickens JR, Wilson CJ (1998) Regulation of action-potential firing in spiny neurons of the rat neostriatum in vivo. J Neurophysiol 79:2358-2364.

Wilson CJ (1993) The generation of natural firing patterns in neostriatal neurons. In: Progress in brain research. Chemical signaling in the basal ganglia (Arbuthnott GW, Emson PC, eds), pp 277-297. Amsterdam: Elsevier.

Wilson CJ (1995) The contribution of cortical neurons to the firing pattern of striatal spiny neurons. In: Models of information processing in the basal ganglia (Houk JC, Davies JL, Beiser DG, eds), pp 29-50. Cambridge, MA: MIT.

Wilson CJ, Groves PM (1981) Spontaneous firing patterns of identified spiny neurons in the rat neostriatum. Brain Res 220:67-80.

Wilson CJ, Kawaguchi Y (1996) The origins of two-state spontaneous membrane potential fluctuations of neostriatal spiny neurons. J Neurosci 16:2397-2410.

Wilson CJ, Chang HT, Kitai ST (1983) Origins of post synaptic potentials evoked in spiny neostriatal projection neurons by thalamic stimulation in the rat. Exp Brain Res 51:217-226. 